\title{
Correlação das espécies colonizadoras com o substrato em áreas abandonadas por mineração
}

\author{
Correlation of colonizing species with substrate in abandoned areas of gold \\ mining
}

\author{
Wander Gladson Amaral ${ }^{I}$, Israel Marinho Pereira ${ }^{\mathrm{II}}$, \\ Evandro Luiz Mendonça Machado ${ }^{\mathrm{II}}$,Cristiany Silva Amaral ${ }^{\mathrm{III}}$, \\ Múcio Magno de Melo Farnezi ${ }^{I I I}$
}

\begin{abstract}
Resumo
A atividade de mineração, importante para a promoção do crescimento econômico e do bem-estar social, tem sido responsável pela modificação de grandes extensões de paisagens do território nacional. Dessa forma, objetivou-se no presente estudo correlacionar às espécies vegetais em três diferentes sítios de uma área de mineração de ouro abandonada há mais de cem anos. A área em estudo foi estratificada em três sítios, sendo que em cada sítio foram alocadas dez parcelas contiguas de $10 \times 10 \mathrm{~m}$ cada, onde foram amostrados e identificados todos os indivíduos arbustivo-arbóreos com $\left(\mathrm{DAS}_{30} \geq 3 \mathrm{~cm}\right)$. Amostras compostas do substrato foram coletadas à profundidade de $0 \mathrm{~cm}$ a $20 \mathrm{~cm}$, em cada uma das trinta parcelas e analisados os teores de areia, silte, argila, matéria orgânica, $\mathrm{pH}, \mathrm{P}, \mathrm{K}^{+}, \mathrm{Ca}^{2+}, \mathrm{Mg}^{2+}, \mathrm{S}, \mathrm{Al}^{3+},(\mathrm{H}+\mathrm{Al}),(\mathrm{V} \%),(\mathrm{SB}), \mathrm{CTC}$ a pH 7, (T), CTC efetiva (t) e (m\%). Foi analisada a influência de variáveis ambientais sobre a densidade das espécies amostradas, utilizando análise de correspondência canônica, verificando a correlação significativa entre elas $(P>0,01)$ pelo teste de MonteCarlo. Foram encontrados 909 indivíduos, pertencentes a 30 famílias botânicas, 64 gêneros e 86 espécies. As variáveis mais fortemente relacionadas com a vegetação foram silte, $\mathrm{pH}$ e V. As espécies Trembleya parviflora, Myrsine guianensi, Trembleya laniflora, Baccharis brachylaenoides, Tibouchina candoleana, Tibouchina mutabilis e Pseudobrickellia angustissima ocorreram de forma mais abundantes em solos com altos valores de $\mathrm{pH}$, saturação por bases e altas proporções de areia, podendo ser consideradas indicadoras desses habitats.
\end{abstract}

Palavras-chaves: Sucessão ecológica; Regeneração natural; Relação espécie-ambiente; Restauração passiva

\begin{abstract}
The mining activity, important for promoting economic growth and social welfare, has been responsible for the modification of large tracts of landscape nationwide. Thus, this work aimed to relate the colonizing species of the area of gold mining with the substrate, in Diamantina, Minas Gerais state. An area under study was stratified into three locations, with ten contiguous plots measuring $10 \times 10 \mathrm{~m}$ each in each location, where all individuals with shrubs with $\left(\mathrm{DAS}_{30} \geq 3 \mathrm{~cm}\right.$ ) were sampled and recorded. Soil composite samples were collected at a depth of $0 \mathrm{~cm}$ to $20 \mathrm{~cm}$ in each of the thirty plots and analyzed the levels of sand, silt, clay, organic matter, $\mathrm{pH}, \mathrm{P}, \mathrm{K}^{+}, \mathrm{Ca}^{2+}, \mathrm{Mg}^{2+}, \mathrm{S}, \mathrm{Al}^{3+},(\mathrm{H}+\mathrm{Al}),(\mathrm{V} \%),(\mathrm{SB}), \mathrm{CTC}$ at $\mathrm{pH} 7,(\mathrm{~T}), \mathrm{CTC}(\mathrm{t})(\mathrm{m} \%)$. The influence of environmental variables on the density of the sampled species was analyzed by using the canonical correspondence analysis, verifying the significant correlation between them $(\mathrm{P}>0.01)$ by the Monte-Carlo test. Nine-hundred and nine (909) individuals were found, belonging to 30 botanical families, 64 genera and 86 species. The variables most strongly related to vegetation were silt, $\mathrm{pH}$ and $\mathrm{V}$. The species Trembleya parviflora, Myrcine guianensi, Trembleya laniflora, Baccharis brachylaenoides, Tibouchina candoleana, Tibouchina mutabilis e Pseudobrickellia angustissima,occurred were more abundant in soils with high $\mathrm{pH}$, base saturation and high proportions of sand may eventually be shown as potential in the recuperation of mined areas with similar conditions to the area.

Keywords: Ecological succession; Natural regeneration; Species-environment relation; Passive restoration

Engenheiro Florestal, Doutorando em Engenharia Florestal, Departamento de Ciências Florestais, Universidade Federal dos Vales do Jequitinhonha e Mucuri, Campus JK, Rod. MGT 367, km 583, 5000, Bairro Alto da Jacuba, CEP 39100-000, Diamantina (MG), Brasil. wander.amaral@yahoo.com. br (ORCID: 0000-0002-8726-9410)

II Engenheiro Florestal, Dr., Professor adjunto, Departamento de Ciências Florestais, Universidade Federal dos Vales do Jequitinhonha e Mucuri, Campus JK, Rod. MGT 367, km 583, 5000, Bairro Alto da Jacuba, CEP 39100-000, Diamantina (MG), Brasil. imarinhopereira@gmail.com (ORCID: 0000-0003-1035-1253) / evandro.machado@ufvjm.edu.br (ORCID: 0000-0002-9301-5257)

III Engenheiro(a) Florestal, Dr(a)., Departamento de Ciências Florestais, Universidade Federal dos Vales do Jequitinhonha e Mucuri, Campus JK, Rod. MGT 367, km 583, 5000, Bairro Alto da Jacuba, CEP 39100-000, Diamantina (MG), Brasil. cristianyamaral@yahoo.com.br (ORCID: 0000-0002-26999487)/ muciomagno@yahoo.com.br (ORCID:0000-0001-8670-1841)
\end{abstract}




\section{Introdução}

A mineração é uma atividade essencial para o desenvolvimento humano e socioeconômico de qualquer nação. Tendo em vista que os recursos minerais são materiais essenciais para as indústrias e produção agrícola (ZHENQ; PEIJUN; JING, 2012). No entanto, muitas paisagens naturais em todo o mundo são severamente alteradas durante o processo de mineração (MACDONALD et al., 2015).

O grau de mudança causado por perturbações promovido pela atividade de mineração comumente é tão grave, que novos ecossistemas são formados nesses locais (LAARMANN et al., 2015), com alterações nas características física, química e microbiológica e perda da resiliência do sítio, o que torna essas áreas de difícil recuperação. Essas áreas na maioria das vezes configuram ambientes sensíveis e importantes para a conservação da biodiversidade, dos recursos hídricos, da paisagem e manutenção dos serviços ecossistêmicos.

Atualmente, têm-se buscado alternativas para a restauração de áreas degradadas que possibilitem a redução dos custos e o retorno dessas áreas a uma condição ecológica mais próxima da original. Nessa nova tendência, têm sido preconizado o manejo e a indução dos processos ecológicos, com vistas a aproveitar ou estimular a resiliência do ecossistema. Para isso, o conhecimento da vegetação colonizadora dessas áreas degradadas, bem como aspectos relacionados às condições edáficas e à autoecologia das espécies, são fundamentais para a definição de metodologias de restauração (CROUZEILLES et al., 2017; BALESTRIN et al., 2019).

Nos Trópicos, a heterogeneidade ambiental influencia diretamente a distribuição e a coexistência de espécies com diferentes exigências às condições microambientais. Estudos mostraram que as características edáficas influenciam a distribuição das espécies arbóreas (CLARK; PALMER; CLARK, 1999). A mesma relação foi observada em trabalhos que relacionam os tipos de solo com fisionomias de cerrado (MENINO et al., 2012) e Mata Atlântica (PEREIRA; OLIVEIRA FILHO; LEMOS-FILHO, 2007). Esses autores observaram que áreas em condições semelhantes de clima, altitude e tipo de solo apresentam maiores similaridades florísticas. Assim, essa variação ambiental, associada à sua localização geográfica responsável por uma grande heterogeneidade na distribuição das espécies, favorece algumas características, como a elevada diversidade em diferentes tipos ecossistemas (PEREIRA; OLIVEIRA FILHO; LEMOSFILHO, 2007; STEIN; KREFT, 2014).

As lavras de mineração de ouro abandonadas apresentam uma elevada heterogeneidade de ambientes em função do processo das condições de relevo e método de exploração do mineral. Nesse sentido, conhecer a vegetação nativa e a sua relação com os solos ou substrato onde ocorrem, além de auxiliar na conservação da biodiversidade local, pode contribuir para orientar os projetos de restauração ecológica. Dessa forma, objetivou-se neste estudo correlacionar às espécies colonizadoras em uma área de mineração de ouro em processo de regeneração natural com o substrato local, e assim, gerar conhecimentos que permitam subsidiar ações de restauração ecológica em ambientes similares.

\section{Materiais e métodos}

\section{Localização e caracterização da área de estudo}

O estudo foi realizado em uma área de mineração de ouro abandonada a mais de cem anos, onde não há relatos de atividades antrópicas posteriores. A área encontra-se localizada no interior do Parque Estadual do Biribiri (PEB), no município de Diamantina, Minas Gerais, na região do Alto Jequitinhonha, no Complexo da Serra do Espinhaço, situado nas coordenadas geográficas $18^{\circ} 23^{\prime} 08^{\prime \prime S ~ e ~} 43^{\circ} 64^{\prime} 78^{\prime \prime} \mathrm{W}$ e altitude média de 1.250 metros. O regime climático da Serra 
do Espinhaço Meridional, região de Diamantina, é tipicamente tropical, Cwb na classificação de Koppen, caracterizado por verões brandos e úmidos (outubro a abril) e invernos mais frescos e secos (junho a agosto). A precipitação média anual varia de 1.250 a $1.550 \mathrm{~mm}$ e a temperatura média anual situa-se na faixa de $18^{\circ}$ a $19^{\circ} \mathrm{C}$, sendo predominantemente amenas durante todo o ano, devido às superfícies mais elevadas dessa serra (VIEIRA et al., 2010). A umidade relativa do ar é quase sempre elevada, revelando médias anuais de 75,6\% (VIEIRA et al., 2010).

As características peculiares do relevo são determinadas, sobretudo, pela natureza quartzítica das rochas que constituem, predominantemente, essa serra, ou seja, seis rochas compostas quase que exclusivamente do mineral quartzo, que além de quase insolúvel, promove a formação de solos arenosos e rasos (ALMEIDA-ABREU; FRAGA; NEVES, 2005).

No entorno da área minerada verifica-se a ocorrência de vegetação típica de Cerrado e algumas manchas de Floresta Estacional Semidecidual. A vegetação colonizadora da área de estudo apresenta elementos da flora das duas tipologias, mas com predomínio do Cerrado. Os solos do Cerrado rupestre são Neossolos Litólicos (EMBRAPA, 2013), originados da decomposição de arenitos e quartzitos, apresentando baixos teores de matéria orgânica e muito pedregosos, o que favorece a desagregação dos elementos e a sua degradação pelos fatores físicos. A perturbação ocorrida na área pela atividade minerária ocasionou a perda da cobertura vegetal, exposição à radiação solar, aumento da temperatura, perda de umidade e dos nutrientes do solo. Porém, a área apresenta um substrato revolvido, com mistura de horizontes de solo (observação de campo).

Para a realização do inventário, a área minerada foi amostrada em três estratos. Sendo estes caracterizados em função do estágio de sucessão ecológica e de sua posição no relevo, assim caracterizados: Sítio 1: Encosta Leste, local com maior proporção de plantas colonizadoras; Sítio 2: Centro da lavra, local com proporção de plantas colonizadoras intermediárias e com pouca presença de samambaias; Sítio 3: Encosta Oeste, local com menor proporção de plantas colonizadoras e alta cobertura da área com samambaias.

\section{Amostragem da Vegetação}

Para a amostragem da vegetação foram plotadas trinta parcelas de $100 \mathrm{~m}^{2}(10 \times 10 \mathrm{~m})$, distribuídas em três sítios (dez parcelas em cada), onde foram inventariados todos os indivíduos arbustivo-arbóreos vivos encontrados no interior das parcelas com DAS30 $\geq 3 \mathrm{~cm}$. A identificação do material botânico foi realizada em campo pelas equipes de coleta e também por meio de consultas à literatura e especialistas ou por comparações com espécimes existentes nos Herbário da Universidade Federal dos Vales do Jequitinhonha e Mucuri (UFVJM). As exsicatas foram depositadas no Herbário Dendrológico Jeanine Felfili, Minas Gerais (HDJF) e as espécies foram classificadas nas famílias reconhecidas pelo sistema do Angiosperm Phylogeny Group IV(ANGIOSPERM PHYLOGENY GROUP, 2016).

\section{Amostragem das variáveis ambientais}

\section{Análise do substrato}

Foi coletada em cada parcela uma amostra composta de substrato na profundidade de 0 a $20 \mathrm{~cm}$; cada amostra composta de substrato $\left(300 \mathrm{~cm}^{3}\right)$ foi constituída de cinco amostras simples. As análises químicas e físicas das amostras foram realizadas no Laboratório de Fertilidade e Física do Solo da UFVJM de acordo com o protocolo de Silva (2009), sendo analisados os parâmetros químicos: $\mathrm{pH}$ em água; teores de $\mathrm{P}, \mathrm{K}, \mathrm{Ca}, \mathrm{Mg}, \mathrm{S}$ e $\mathrm{Al}$; complexo sortivo (acidez potencial $(\mathrm{H}+\mathrm{Al}$ ), saturação por bases (V\%), soma de bases (SB), CTCa pH 7 (T), CTC efetiva (t) e saturação por alumínio (m\%)) e matéria orgânica (MO). De acordo com a EMBRAPA (1997), foram analisados a granulometria (\% de areia, \% de silte e \% de argila) das amostras. 
Análise dos dados

Para testar a hipótese da existência de relação entre a estrutura e a composição florística da vegetação colonizadora em uma área de mineração de ouro, com fatores ambientais edáficos, foi utilizada a ordenação das parcelas, espécies e variáveis ambientais pelo método de Análise de Correspondência Canônica (TER BRAAK; PRENTICE, 1988). Esse diagrama possibilita a visualização de um padrão de variação da comunidade, bem como das características principais responsáveis pelas distribuições das espécies ao longo das variáveis ambientais.

Os dados dos atributos químicos e físicos do substrato dos sítios de estudo foram submetidos à análise de componentes principais (ACP), com o objetivo de verificar quais os parâmetros químicos (13 variáveis) e físicos (3 variáveis) poderiam ser utilizados para diferenciar ou para indicar similaridades entre as amostras de substratos dos sítios estudados.

Para a CCA, foram organizadas as duas matrizes, sendo que a matriz de abundância das espécies foi constituída do número de indivíduos por parcela da i-ésima espécie. Foram utilizadas apenas as espécies que apresentaram cinco ou mais indivíduos na amostragem. Esse procedimento é considerado conveniente nas técnicas de ordenação em geral, pois espécies raras ou de baixa densidade aumentam o volume de cálculo e os erros de interpretação. De acordo com as recomendações de Ter Braak (1995), os valores de abundância foram transformados pela expressão $\log 10(\mathrm{a}+1)$ para compensar os desvios causados por alguns valores muito elevados. A matriz de variáveis ambientais foi composta por 17 variáveis. Após realizar uma CCA preliminar, com as 17 variáveis estabelecidas inicialmente, foram eliminadas aquelas variáveis ambientais fracamente correlacionadas ou altamente redundantes entre si (PIRES et al., 2014). Para a lavra degradada pela mineração, a CCA final foi processada com as cinco variáveis mais representativas e mais fortemente correlacionadas com os eixos de ordenação, sendo elas: pH, saturação por bases $(\mathrm{V})$, teor de alumínio (Al), matéria orgânica (M.O) e porcentagem de areia.

Para testar a probabilidade de acerto das relações encontradas entre a matriz ambiental, composta por fatores edáficos, e a matriz de espécies, empregou-se o teste de permutação de "Monte Carlo". As análises foram efetuadas, utilizando o programa PC-ORD versão 4.14 (MCCUNE; MEFFORD, 1997).

\section{Resultados e discussão}

Os substratos apresentam baixa fertilidade natural, com saturação de bases inferior a $50 \%$ (distróficos), muito baixos teores de P e K, e com altos teores de alumínio e acidez elevada. Resultado semelhante foi encontrado por Martins et al. (2003), ao estudarem a distribuição das espécies arbóreas em virtude do gradiente topográfico em uma floresta estacional semidecidual em Viçosa. A classe textural foi predominantemente franco arenoso para os sítios 1 e 2 , e predominantemente franco siltoso no sítio 3 (Tabela 1). Já os teores de matéria orgânica para os três sítios variaram de médio a baixo, minimizando a disponibilidade de nutrientes para as plantas, dificultando assim o seu estabelecimento na área. Há uma tendência, esperada, de ocorrerem maiores teores de acidez total $(\mathrm{H}+\mathrm{Al})$ em solos mais ricos em matéria orgânica, principalmente se estes apresentarem pH baixo (CAMARGO et al., 2008). Esse fato foi comprovado analisando os teores de $(\mathrm{H}+\mathrm{Al})$ no sítio I, onde os altos teores de M.O são mais evidentes. Benites et al. (2007), em estudo com solos associados a afloramentos rochosos na serra da Mantiqueira e Espinhaço, encontraram solos invariavelmente com baixo teor de nutrientes, níveis elevados de alumínio trocável e horizontes escuros devido ao acúmulo de matéria orgânica (BENITES et al., 2007).

$\mathrm{Na}$ área de estudo, as espécies arbustivo-arbóreas se desenvolvem em substrato muito ácido (entre 4,3 e 4,6) e com teores de matéria orgânica considerados muito baixa (entre 1,8\% e $4,2 \%$ ) (Tabela 1). Sabe-se que as plantas dos campos rupestres vivem sobre solos arenosos ou pedregosos, podendo também ocorrer diretamente sobre as rochas. Esses padrões de baixa 
fertilidade, alta acidez e excesso de alumínio são comuns nas áreas de cerrado (FELFILI; SILVA JÚNIOR, 2005). No entanto, apesar dos solos serem rasos, arenosos ou até mesmo pedregosos, pobres em nutrientes e ácidos, há uma extraordinária variedade de espécies que são adaptadas a essas condições, devido a apresentarem características ecofisiológicas e morfológicas que permitem suas sobrevivências sobre essas condições (OTONI et al., 2013). Além disso, existem períodos de absoluta carência de água, uma vez que, no Espinhaço Meridional, há uma estação seca bem definida, ficando as plantas sujeitas ao estresse hídrico por aproximadamente cinco meses (abril a agosto) (VIEIRA et al., 2010).

Tabela 1 - Valores médios e desvio-padrão dos atributos químicos e físicos dos substratos nos diferentes sítios da área de estudo no PEB, em Diamantina, MG

Table 1 - Mean values and standard deviation of the chemical and physical attributes of the substrates in the different sites of the study area at PEB, in Diamantina, MG state

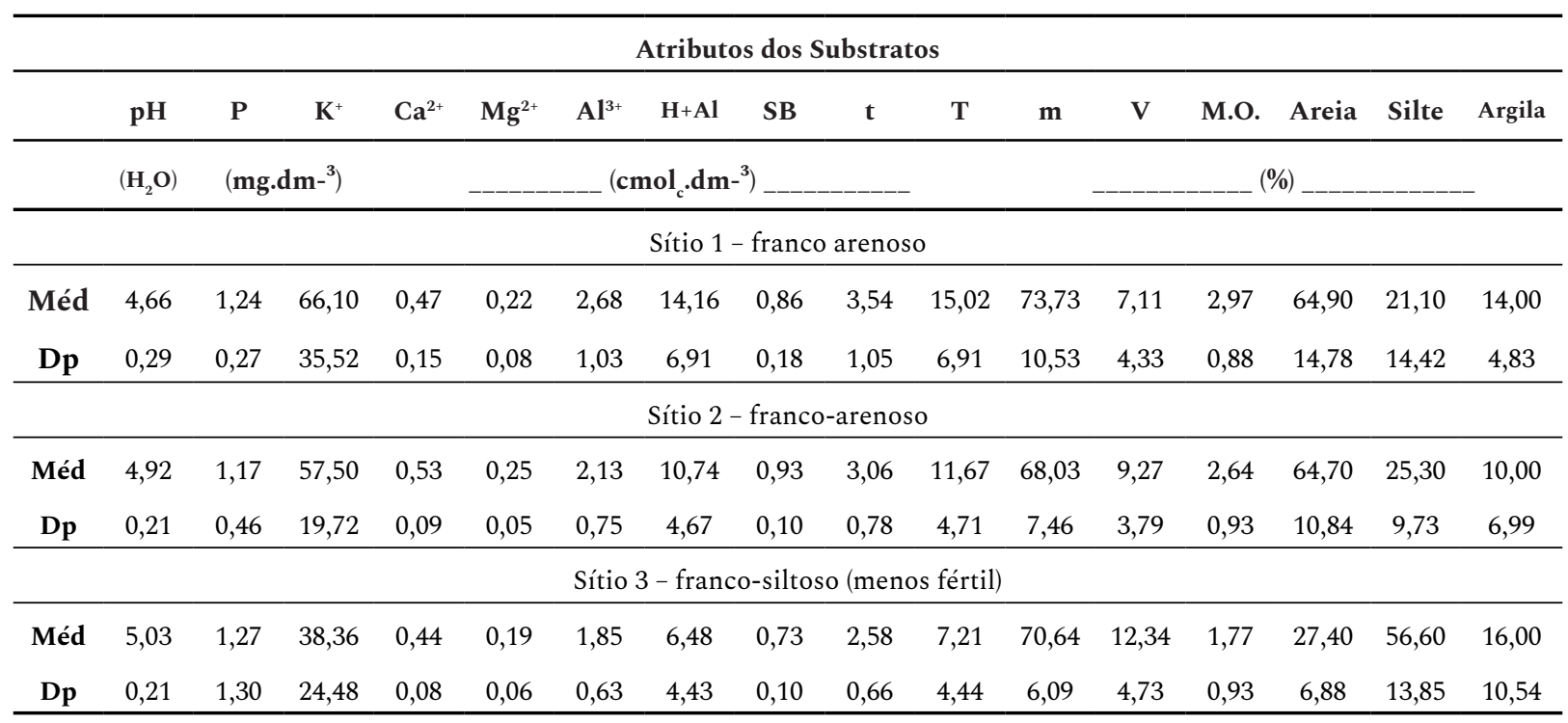

Fonte: Autores (2019)

Em que: Méd = valores médios; $\mathrm{Dp}=$ desvio-padrão; $\mathrm{P}$ = teor de fósforo disponível; $\mathrm{K}=$ teor de potássio disponível; $\mathrm{Ca}^{2+}=$ teor de cálcio disponível; $\mathrm{Mg}^{2+}=$ teor de magnésio disponível; $\mathrm{Al}^{3+}=$ teor de alumínio; $\mathrm{H}+\mathrm{Al}=$ acidez potencial; $\mathrm{SB}=$ soma de bases trocáveis; $\mathrm{t}=$ capacidade de troca de cátions efetiva; $\mathrm{T}$ = capacidade de troca de cátions a pH7; $\mathrm{m}$ = saturação por alumínio; $\mathrm{V}$ = saturação por bases; M.O. = matéria orgânica.

Verifica-se, na Figura 1, que houve a formação de dois grupos distintos, sendo que, no primeiro, estão praticamente todas as parcelas do sítio 3, localizadas sobre solos que apresentam maiores porcentagens de silte e argila juntamente com maiores valores de fósforo (P), pH e alta saturação por base. As parcelas dos sítios 1 e 2 formaram o segundo grupo, não havendo distinção dos mesmos em função das variáveis ambientais, o que pode explicar a semelhança deles com relação ao número de espécies e número de indivíduos.

Nos três sítios, uma característica comum do substrato é apresentar saturação por base inferior a 50\%. E com isso, Balduino et al. (2005), estudando a flora lenhosa do Cerrado stricto sensu em áreas nativas, relatam que as famílias Fabaceae,Vochysiaceae, Myrtaceae, Malpighiaceae e Rubiaceae apresentam os mais altos valores de riqueza florística quando presentes em solos distróficos. O que pode explicar a ocorrência de tais famílias como sendo as mais importantes da flora estudada. 
Figura 1 - Análise de componentes principais das variáveis ambientais coletadas em uma área degradada em processo de regeneração natural em Diamantina, MG

Figure 1 - Principal components analysis of environmental variables collected in a degraded area in process of natural regeneration in Diamantina, MG state

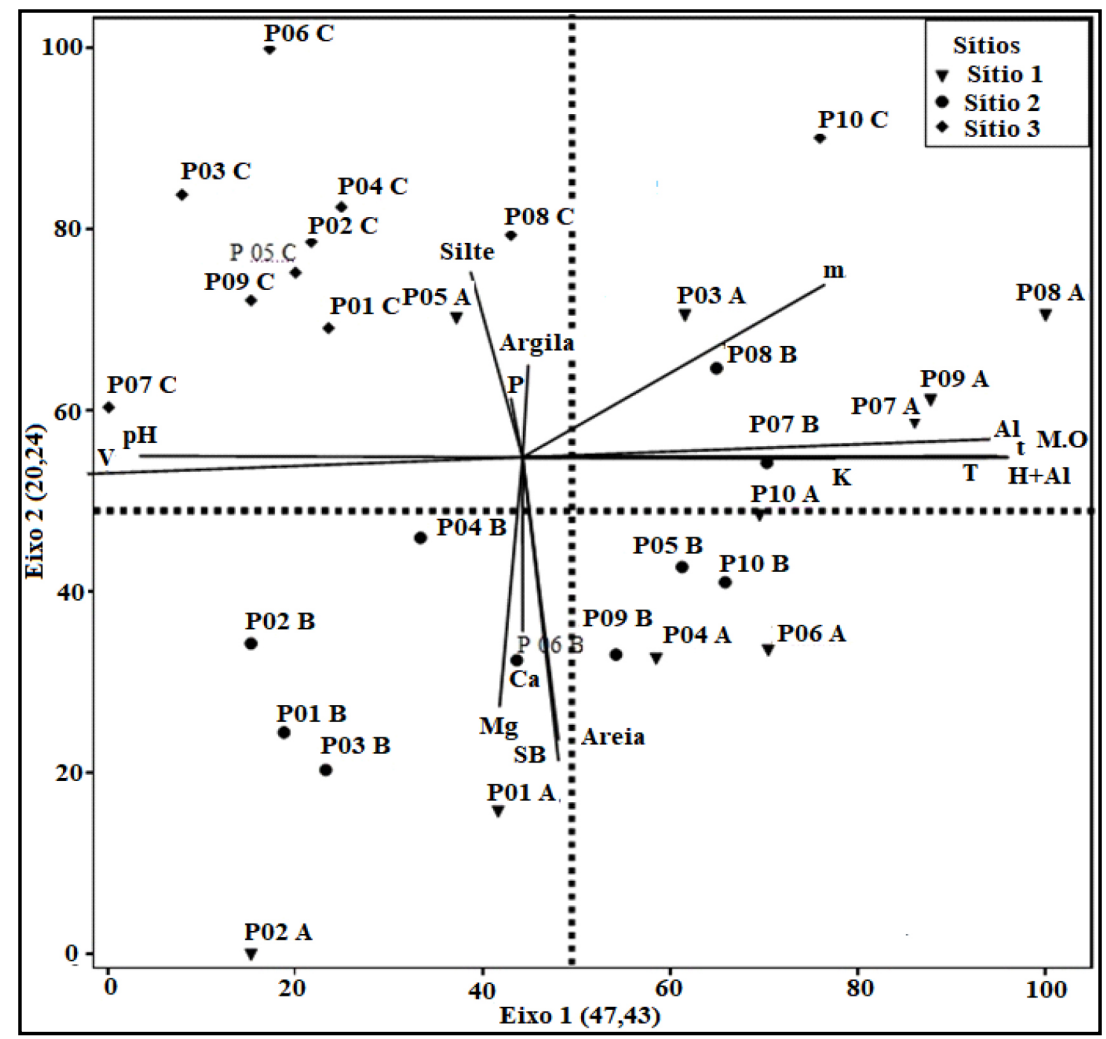

Fonte: Autores (2019)

Foram inventariados 909 indivíduos, pertencentes a 30 famílias, 64 gêneros e 86 espécies (Tabela 2). Valores próximos foram encontrados por Felfili (2001), estudando uma área de Cerrado Stricto Sensu em Brasília (DF), onde foram encontradas 34 famílias botânicas contendo 60 gêneros e 80 espécies. As famílias com maior número de espécies foram: Myrtaceae (18); Melastomataceae (10); Asteraceae (8); Fabaceae (6); Rubiacae (5); Aquifoliaceae (4) e Malpighiaceae (4). Essas famílias, em termos de indivíduos, representaram $67,33 \%$ do total de indivíduos amostrados. Resultados semelhantes foram registrados por Corrêa, Mélo Filho e Batista (2007), estudando o estrato lenhoso em áreas mineradas no Distrito Federal.

Esse padrão de distribuição, tanto das famílias como dos gêneros, é frequente na literatura, onde se observa diminuição da contribuição da família Fabaceae à medida que o gradiente altitudinal aumenta (PEREIRA et al., 2006). Contudo, padrão inverso é observado para Lauraceae e Myrtaceae, cuja contribuição aumenta ao passo que a altitude se torna mais elevada. Dessa forma, quando se analisa em nível de gênero, Eremanthus, Myrsine e Ocotea são gêneros que tendem a aumentar sua abundância à medida que a altitude se torna mais elevada (PEREIRA et al., 2006). 
Tabela 2 - Relação das espécies arbustivo-arbóreas registradas em uma área abandonada pela mineração de ouro no PEB, Diamantina-MG, dispostas em ordem alfabética de família, seguidas de seus respectivos números de indivíduos

Table 2 - List of woody species recorded in an area abandoned for gold mining at PEB, Diamantina-MG state, arranged in alphabetical order by family, followed by their number of individuals

\begin{tabular}{|c|c|c|c|c|c|c|c|c|c|}
\hline \multirow{2}{*}{ Família/Espécies } & \multicolumn{4}{|c|}{ Sítio (NI) } & \multirow{2}{*}{ Família/Espécies } & \multicolumn{4}{|c|}{ Sítio (NI) } \\
\hline & 1 & 2 & 3 & $\mathbf{T}$ & & 1 & 2 & 3 & $\mathbf{T}$ \\
\hline $\begin{array}{l}\text { ANACARDIACEAE } \\
\text { Tapirira obtusa (Benth.) J.D.Mitch. } \\
\text { ANNONACEAE }\end{array}$ & 1 & - & - & 1 & $\begin{array}{l}\text { MONIMIACEAE } \\
\text { Mollinedia argyrogyna Perkins. } \\
\text { MYRSINACEAE }\end{array}$ & 1 & - & - & 1 \\
\hline \multirow{2}{*}{ Família/Espécies } & \multicolumn{4}{|c|}{ Sítio (NI) } & \multirow{2}{*}{ Família/Espécies } & \multicolumn{4}{|c|}{ Sítio (NI) } \\
\hline & 1 & 2 & 3 & $\mathbf{T}$ & & 1 & 2 & 3 & $\mathbf{T}$ \\
\hline Xylopia sericea A.St.-Hil. & 9 & 8 & 3 & 20 & Myrcine ferruginea A. AC. & - & 1 & - & 1 \\
\hline Ilex affinis Gardn. & 1 & - & 2 & 3 & Myrsine coriacea $(\mathrm{Sw}$.$) .$ & 3 & 1 & 27 & 31 \\
\hline Ilex brevicuspis Reissek. & - & 1 & - & 1 & Myrsine gardneriana A.DC. & - & 1 & - & 1 \\
\hline Ilex conocarpa Reissek & 2 & - & - & 2 & Myrsine guianensis (Aubl.) Kuntze. & 1 & 1 & - & 2 \\
\hline Ilex theezans Mart. ex Reissek. & - & - & 2 & 2 & MYRTACEAE & & & & \\
\hline ASTERACEAE & & & & & Calyptranthes widgreniana O.Berg. & 1 & - & - & 1 \\
\hline Austrocritonia velutina (Gardn.). & 1 & - & - & 1 & Eugenia glazioviana Kiaersk. & 1 & - & - & 1 \\
\hline Baccharis brachylaenoides DC. & 2 & 16 & - & 18 & Marlierea laevigata (DC.) Kiaersk. & - & 9 & 1 & 10 \\
\hline Baccharis dentata (Vell.) G.M.Barroso. & 9 & 3 & 7 & 19 & Myrceugenia ovalifolia (O.Berg). & - & 2 & 1 & 3 \\
\hline Eremanthus erytropappus. & - & 1 & - & 1 & Myrcia amazonica DC. & 1 & 1 & - & 2 \\
\hline Eremanthus incanus (Less.). & 74 & 72 & 14 & 160 & Myrcia guianensis (Aubl.) DC. & - & 7 & - & 7 \\
\hline Lychnophora pohlli mart. & - & 13 & 1 & 14 & Myrcia hartwegiana (O.Berg) Kiaersk & - & 3 & - & 3 \\
\hline Pseudobrickellia angustissima R.M.King & 12 & - & - & 12 & Myrcia mischophylla Kiaersk. & - & 4 & - & 4 \\
\hline Vernonanthura phosphorica (Vell.) & 1 & - & - & 1 & Myrcia mutabilis (O.Berg) N.Silveira. & - & 7 & 2 & 9 \\
\hline BIGNONIACEAE & & & & & Myrcia pulchra (O.Berg) Kiaersk. & - & 1 & - & 1 \\
\hline Zeyheria montana Mart. & 1 & - & - & 1 & Myrcia retorta Cambess. & 1 & - & - & 1 \\
\hline BURSERACEAE & & & & & Myrcia splendens (Sw.) DC. & 2 & - & - & 2 \\
\hline Protium brasiliense (Spreng.) Engl. & - & 2 & - & 2 & Myrcia venulosa DC. & 1 & 2 & - & 3 \\
\hline CLUSIACEAE & & & & & Psidium cattleianum Afzel. ex Sabine. & 3 & - & - & 3 \\
\hline Kielmeyera lathrophyton Saddi. & - & 1 & - & 1 & Siphoneugena densiflora O.Berg. & - & 2 & - & 2 \\
\hline ERYTHROXYLACEAE & & & & & Sp. 1 & 2 & 1 & 2 & 5 \\
\hline Erythroxylum cuneifolium (Mart.). & 2 & - & - & 2 & & & & & \\
\hline EUPHORBIACEAE & & & & & NYCTAGINACEAE & & & & \\
\hline Pera glabrata (Schott) Poepp. ex Baill. & 1 & 1 & - & 2 & Guapira hirsuta (Choisy) Lundell. & 2 & - & - & 2 \\
\hline Sapium glandulosum (L.) Morong. & - & 2 & - & 2 & Guapira opposita (Vell.) Reitz. & 18 & 11 & 5 & 34 \\
\hline
\end{tabular}


Tabela 1 - Conclusão ...

Table 1 - Conclusion ...

\begin{tabular}{|c|c|c|c|c|c|c|c|c|c|}
\hline \multirow{2}{*}{ Família/Espécies } & \multicolumn{4}{|c|}{ Sítio (NI) } & \multirow{2}{*}{ Família/Espécies } & \multicolumn{4}{|c|}{ Sítio $(\mathrm{NI})$} \\
\hline & 1 & 2 & 3 & $\mathbf{T}$ & & 1 & 2 & 3 & $\mathbf{T}$ \\
\hline FABACEAE & & & & & POLYGONACEAE & & & & \\
\hline Copaifera langsdorffii Desf. & 1 & - & - & 1 & Coccoloba brasiliensis Nees \& Mart. & 59 & 15 & 6 & 80 \\
\hline Senna aristeguietae H.S.Irwin \& Barneby & 16 & 1 & - & 17 & PROTEACEAE & & & & \\
\hline Tachigali rugosa (Mart. ex Benth.) & 8 & 3 & - & 11 & Roupala montana Aubl. & 27 & 3 & 6 & 36 \\
\hline Diplotropis ferruginea Benth. & 1 & - & - & 1 & RUBIACEAE & & & & \\
\hline Swartzia apetala Raddi. & 3 & 2 & - & 5 & Bathysa cuspidata (A.St.-Hil.) Hook.f. & 1 & - & - & 1 \\
\hline Stryphnodendron polyphyllum Mart. & 1 & - & 1 & 2 & Cordiera sessilis (Vell.) Kuntze. & 6 & 9 & 2 & 17 \\
\hline LAURACEAE & & & & & Coussarea hydrangeifolia (Benth.). & 1 & - & - & 1 \\
\hline Nectandra sp. & 1 & - & - & 1 & Coutarea hexandra (Jacq.) K.Schum. & 2 & - & - & 2 \\
\hline Ocotea lancifolia (Schott) Mez. & 5 & 1 & - & 6 & Palicoureamacrobotrys (Ruiz \& Pav.) & 1 & - & - & 1 \\
\hline MALPIGHIACEAE & & & & & SALICACEAE & & & & \\
\hline Banisteriopsis muricata (Cav.) Cuatrec. & 2 & - & - & 2 & Casearia rupestris Eichler & 1 & - & - & 1 \\
\hline Byrsonima coccolobifolia Kunth. & 4 & - & - & 4 & SAPINDACEAE & & & & \\
\hline Byrsonima crassa Nied. & - & 3 & - & 3 & Averrhoidium garnerianum Baill. & 1 & 1 & - & 2 \\
\hline Heteropterys byrsonimifolia A.Juss. & 1 & - & - & 1 & Dodonaea viscosa Jacq. & - & 2 & 2 & 4 \\
\hline MELASTOMATACEAE & & & & & SAPOTACEAE & & & & \\
\hline Lavoisiera montana Cogn. & - & - & 11 & 11 & Micropholis gardneriana (A.DC.). & - & - & 1 & 1 \\
\hline Lavoisiera pectinata Cogn. & 1 & 3 & - & 4 & SIMAROUBACEAE & & & & \\
\hline Miconia sp. & 1 & - & - & 1 & Simarouba versicolor A.St.-Hil. & 11 & 4 & 3 & 18 \\
\hline Miconia pepericarpa DC. & 3 & 1 & 3 & 7 & STYRACACEAE & & & & \\
\hline Microlicia isophylla DC. & 2 & - & - & 2 & Styrax camporus Pohl. & 1 & - & - & 1 \\
\hline Tibouchina candolleana (Mart.). & 2 & 43 & 4 & 49 & THEACEAE & & & & \\
\hline Tibouchina mutabilis (Vell.) Cong. & 7 & 1 & 1 & 9 & Laplacea tomentosa (Mart. \& Zucc.). & 3 & - & 2 & 5 \\
\hline Trembleia sp1. & 3 & 1 & - & 4 & VERBENACEAE & & & & \\
\hline Trembleya parviflora (D.Don) Cogn. & - & 44 & - & 44 & Lippia sp1. & 5 & - & - & 5 \\
\hline Trembleya laniflora (D. Don) Cogn. & 12 & 7 & 39 & 58 & Lippia sp2. & 1 & - & - & 1 \\
\hline Vochisia maxima. & 1 & - & - & 1 & VOCHYSIACEAE & & & & \\
\hline MELIACEAE & & & & & Vochysia tucanorum Mart. & - & 1 & - & 1 \\
\hline Cabralea canjerana (Vell.) Mart. & - & 2 & - & 2 & Total & 157 & 89 & 60 & 306 \\
\hline
\end{tabular}

Fonte: Autores (2019)

Em que: $\mathrm{T}=$ total e $\mathrm{NI}=$ número de indivíduos.

Os resultados da análise de correspondência canônica (CCA) estão representados nos diagramas de ordenação das Figuras 2A e 2B. Na análise dos padrões florísticos e ambientais, as CCAs apresentaram autovalores muito baixos para os dois primeiros eixos, com o primeiro eixo explicando 11,7\% (Eixo 1) e o segundo 8,8\% (Eixo 2) da variância global dos dados (total acumulado: $20,5 \%$ ), indicando a presença de relativamente muito 'ruído' ou variância remanescente não explicada (79,5\%). Os gradientes são curtos (< 0,5 sensu, TER BRAAK, 1995), ou seja, há grande número de espécies distribuídas ao longo dos gradientes, porém variando sua abundância relativa (TER BRAAK, 1995). Sendo assim, não se pode afirmar, no presente caso, que a distribuição das 
espécies na lavra abandonada esteja condicionada, primariamente, por características químicas e físicas do solo. O gradiente catenário, da esquerda para a direita, envolve teor de alumínio (Al), teor de matéria orgânica (M.O), porcentagem de areia, pH e saturação por base.

Figura 2 - Diagrama de ordenação das parcelas $(\mathrm{A})$ e espécies $(\mathrm{B})$ produzidos pela análise de correspondência canônica (CCA) da distribuição do número de indivíduos de 28 espécies arbóreas em três sítios de uma área degradada em processo de regeneração natural em Diamantina, MG

Figure 2 - Diagram of the ordination of plots (A) and species (B) produced by the canonical correspondence analysis (CCA) of the distribution of the number of individuals of 28 tree species in three environments of a degraded area in the process of natural regeneration in

Diamantina, MG state

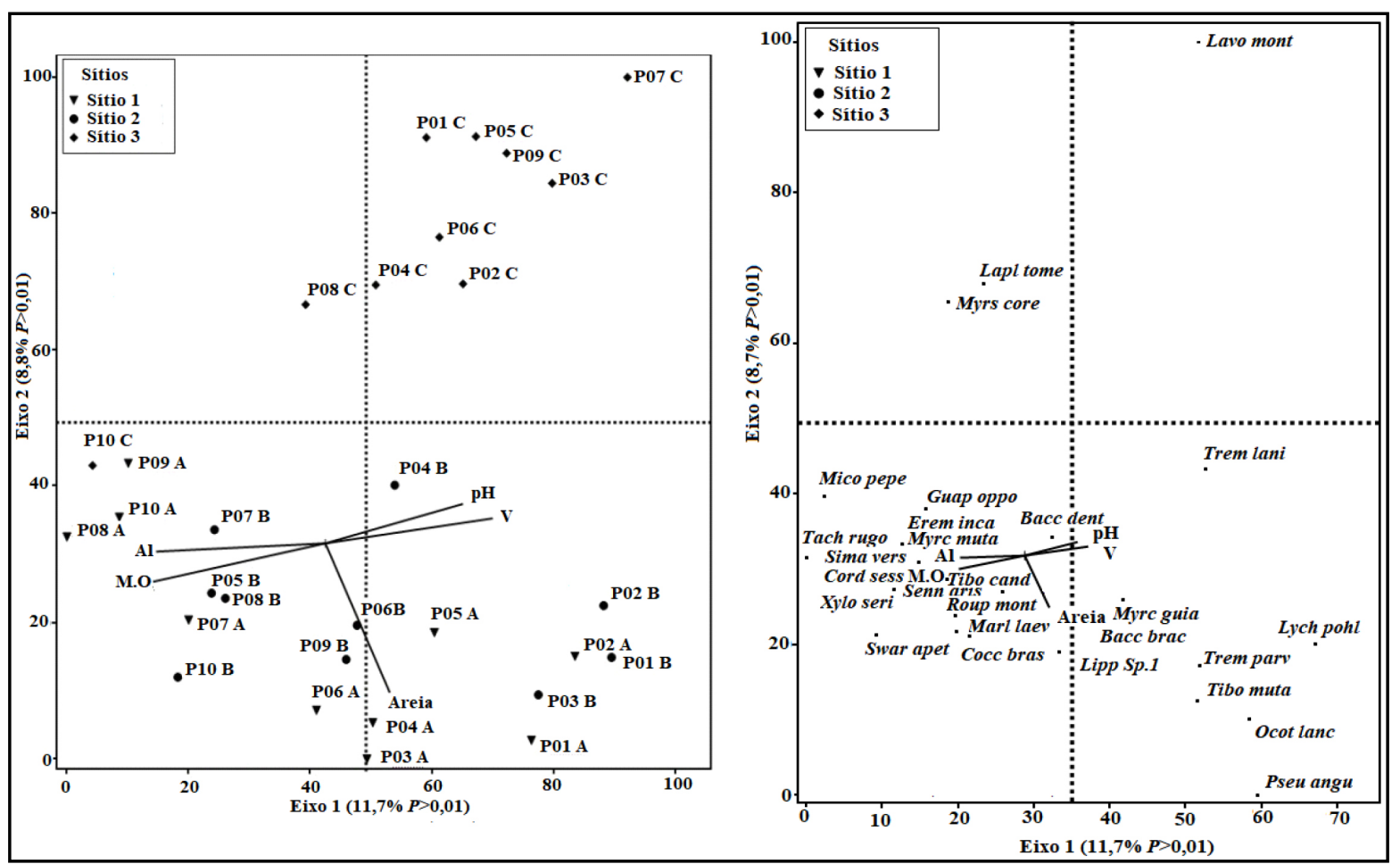

Fonte: Autores (2019)

Estudos sobre dinâmica de clareiras consideram que eventos biológicos ocorridos na história de cada sítio não podem ser desconsiderados, quando se tenta explicar padrões de distribuição espacial de árvores em florestas tropicais (AUBERT; OLIVEIRA-FILHO, 1994). Mesmo assim, de acordo com Ter Braak e Prentice (1988), baixos valores da variância para a abundância das espécies são comuns em dados de vegetação e não prejudicam a significância das relações espécie-ambiente. Além disso, o teste de permutação de Monte Carlo indicou que as abundâncias das espécies e variáveis ambientais foram significativamente correlacionadas com primeiro eixo de ordenação $(\mathrm{P}>0,01)$. Variáveis fundamentais, como luz, água e fatores de dispersão das espécies, nem sempre são facilmente perceptíveis ou mensuráveis (BOTREL et al.,2002), o que ressalta a necessidade de muita cautela na interpretação de qualquer resultado de correlações efetuadas entre a distribuição das espécies e variáveis do ambiente físico. Além disso, as espécies de uma comunidade respondem de forma interativa, e não isolada, às condições do sítio (BOTREL et al., 2002), evidenciando a complexidade da relação solo-planta.

Ter Braak e Prentice (1988) afirmam que perturbações não-explicadas pela CCA são vistas 
como naturais e não comprometem a significância das correlações encontradas. Resultados semelhantes foram encontrados por Nappo, Oliveira-Filho e Martins (2000), estudando a estrutura do sub-bosque de povoamentos homogêneos de Mimosascabrella Bentham, em outra área minerada de Minas Gerais. Para a área de mineração de ouro abandonada a mais de cem anos no PEB, as variáveis ambientais mais fortemente correlacionadas com o primeiro eixo de ordenação foram $\mathrm{pH}$, saturação por bases $(\mathrm{V})$, alumínio $\left(\mathrm{Al}^{3+}\right)$, matéria orgânica (M.O) e porcentagem de areia. Nesse contexto, a ordenação das parcelas no primeiro eixo (Figura 2A) sugere, claramente, uma forte separação entre as parcelas do sítio 3, indo ao encontro de altos valores de pH e saturação por bases e silte (veja PCA e Tabela 1). Já as parcelas dos sítios 1 e 2 se misturaram em função de todas as variáveis ambientais relatadas acima.

$\mathrm{Na}$ ordenação das espécies pela CCA (Figura 2B), verifica-se claramente a formação de três grupos distintos. O primeiro é formado por espécies Trembleya parviflora, Myrcine guianensis, Pseudobrickellia angustissima, Trembleya laniflora, Baccharis brachylaenoides, Lippia sp.1, Tibouchina candoleana, Tibouchina mutabilis, Ocotea lancifolia, Pseudobrickellia angustissima, que ocorreram de forma mais abundantes nas parcelas sobre substratos com maiores valores de $\mathrm{pH}$, saturação por bases e altas proporções de areia. Já o segundo grupo é formado pelas espécies, Roupala Montana, Marlierea laevigata, Coccoloba brasiliensis, Swartza apelata, Senna aristeguietae, Xilopia sericea, Cordieres sesilis, Simarouba versicolor, Tachigali rugosa, Myrcia multiflora, Eremanthus incanus, Guapira opposita, Moconia pepericarpa, as quais ocorreram de forma mais abundante nas parcelas sobre substratos que apresentavam teores mais elevados de alumínio $\left(\mathrm{Al}^{3+}\right)$ e matéria orgânica. O terceiro grupo foi formado pelas espécies Laplaceae tomentosa e Myrcine coreace, as quais aparecem com maior abundância em parcelas sobre substratos com menor porcentagem de areia. Pode-se observar, também na figura 2, que a espécie Lavoisiera montana pode ser indicativo de solos ácidos.

Em áreas naturais contínuas, as variações são geralmente graduais, mas em áreas antropizadas podem ser incluídas uma considerável variação de condições ambientais e, conseqüentemente, de estrutura da comunidade arbórea. Entretanto, cada fragmento pode conter várias peças de mosaico de vegetação relacionadas a diferenças de substrato, à fase sucessional e ou aos efeitos antrópicos. Dessa maneira, conclusões sobre os padrões de distribuição de espécies face as variáveis ambientais só devem se aproximar de uma generalização após muitas repetições do mesmo padrão em diversas áreas.

\section{Conclusão}

A mineração afetou de forma duradoura o processo de sucessão natural na área de estudo. Observou-se que mesmo após um período de mais de cem anos não foi suficiente para o restabelecimento completo da vegetação, sendo detectado variações florísticas entres os sítios outrora impactados. Entretanto, observou-se padrões comuns como fatores ambientais (edáficos) que melhor determinam a distribuição das espécies como é caso dos teores de $\mathrm{pH}$ e as proporções de silte e saturação de bases (V).

Algumas espécies carecem ser melhor investigadas, a saber:Trembleya parviflora, Myrcine guianensis, Lychnophora pohlli, Tibouchina mutabilis, Baccharis brachylaenoides, Lippia sp., Tibouchina candoleana, Trembleya laniflora, Ocotea lancifolia, Pseudobrickellia angustissima, pois ocorreram de forma mais abundantes em substratos com maiores valores de $\mathrm{pH}$, saturação por bases e porcentagem de areia, podendo vir a ser indicadas como potenciais na recuperação de áreas mineradas em condições similares às da área estudada. 


\section{Referências}

ALMEIDA-ABREU, P. A.; FRAGA, L. M. S.; NEVES, S. C. Geologia. In: SILVA, A. C.; PEDREIRA, L. C. V. S. F.; ALMEIDA-ABREU, P. A. (org.). Serra do Espinhaço Meridional: paisagens e ambientes. 1. ed. Diamantina: Faculdade de Ciências Agrárias, 2005. p. 17-44.

ANGIOSPERM PHYLOGENY GROUP. An update of the angiosperm phylogeny group classification for the orders and families of flowering plants: APG IV. Botanical Journal of the Linnean Society, London, v. 141, n. 4, p. 399-436, apr. 2016.

AUBERT, E.; OLIVEIRA-FILHO, A. T. Análise multivariada da estrutura fitossociológica do sub-bosque de plantios experimentais de Eucalyotus spp. e Pinus spp. em Lavras (MG). Revista Árvore, Viçosa, MG, v. 18, n. 3, p. 194-214, 1994.

BALDUINO, A. P. C. et al. Fitossociologia e análise comparativa da composição florística do Cerrado da Flora de Paraopeba - MG. Revista Árvore, Viçosa, MG, v. 29, n. 1, p. 25-34, jan./fev. 2005.

BALESTRIN, D. et al.Phytosociological study to define restoration measures in a mined area in Minas Gerais, Brazil. Ecological Engineering, [s. l.], v. 135, p. 8-16, sept. 2019.

BENITES, V. M. et al. Soils associated with rock outcrops in the Brazilian mountain ranges Mantiqueira and Espinhaço. Revista Brasileira de Botânica, São Paulo, v. 30, n. 4, p. 596-577, 2007.

BOTREL, R. T. et al. Influência do solo e topografia sobre as variações da composição florística e estrutura da comunidade arbórea-arbustiva de uma floresta estacional semidecidual em Ingaí, MG. Revista Brasileira de Botânica, São Paulo, v. 25, n. 2, p. 195-213, jun. 2002.

CAMARGOS, V. L. de et al. V. Influência de fatores edáficos sobre variações floristicas na floresta Estacional Semidecídua no entorno da Lagoa Carioca, Parque estadual do Rio Doce, MG, Brasil. Acta Botânica Brasílica, Belo Horizonte, v. 22, n. 1, p. 75-84, jan./mar. 2008.

CLARK, D. B.; PALMER, M. W.; CLARK, D. A. Edaphic factors and the landscale-scale distributions of tropical rain forest trees. Ecology, California, v. 80, n. 8, p. 2662-2675, dec. 1999.

CORRÊA, R. S.; MÉLO FILHO, B.; BATISTA, G. M. M. Avaliação fitossociológica da sucessão autogênica em áreas mineradas no Distrito Federal. Cerne, Lavras, v. 13, n. 4, p. 406- 415, 2007.

CROUZEILLES, R. et al. Ecological restoration success is higher for natural regeneration than for active restoration in tropical forests. Applied Ecology, [s. l.], v. 3, n. 11, p. 1-7, nov. 2017.

EMBRAPA. Centro Nacional de Pesquisa de Solos. Manual de métodos de análise de solo. Brasília: Produção de Informação, 1997. 212 p.

EMBRAPA. Sistema brasileiro de classificação de solos. 3. ed. Rio de Janeiro: Centro Nacional de Pesquisa de Solos, 2013.

FELFILI, J. M. Fitossocilogia da vegetação arbórea. In: FELFILI, J. M.; SILVA JÚNIOR, M. C. (Org.). Biogeografia do Bioma Cerrado: estudo fitofisionômico na Chapada do Espigão Mestre do São Francisco. Brasília: Universidade de Brasília, 152p, 2001.

FELFILI, J. M.; SILVA JUNIOR, M. C. Diversidade alfa e beta no cerrado sensu stricto, Distrito Federal, Goiás, Minas Gerais e Bahia. In: SCARIOT, A.; SOUSA-SILVA, J. C.; FELFILI, J. M. Cerrado: ecologia, biodiversidade e conservação. Brasília: Ministério do Meio Ambiente, 2005. p. $141-154$.

LAARMANN, D. et al.Evaluation of afforestation development and natural colonization on a reclaimed mine site. Restoration Ecology, Malden, v. 23, n. 3, p. 301-309, feb. 2015.

MACDONALD, S. E. et al. Forest restoration following surface mining disturbance: challenges 
and solutions. New Forests, Dordrecht, v. 46, p. 703-732, nov. 2015.

MARTINS, S. V. et al. Distribuição de espécies arbóreas em um gradiente topográfico de floresta estacional semidecidual em Viçosa, MG. Scientia Forestalis, Piracicaba, v. 2, n. 64, p. 172-181, dez. 2003.

MCCUNE, B.; MEFFORD, M. J. PC-ORD for Windows: Multivariate Analysis of ecological data - version 3.12. Oregon: MJM Software Design, 1997.

MENINO, G. C. O. et al. Environmental heterogeneity and natural regene ration in riparian vegetation of the Brazilian Semi-Arid Region. Edinburgh Journal of Botany, [s. l.], v. 69, n. 1, p. 29-51, mar. 2012.

NAPPO, M. E.; OLIVEIRA-FILHO, A. T.; MARTINS, S. V. A estrutura do sub-bosque de povoamentos homogêneos de Mimosa scabrella Bentham, em área minerada, em Poços de Caldas, MG. Ciência Florestal, Santa Maria, v. 10, n. 2, p. 17-29, jul./dez. 2000.

OTONI, T. J. O. et al. Componente arbóreo, estrutura fitossociológica e relações ambientais em um remanescente de cerradão, em Curvelo-MG. Cerne, v. 19, n. 2, p. 201-211, abr./june 2013.

PEREIRA, I. M. et al. Composição florística do compartimento arbóreo de cinco remanescentes florestais do maciço do Itatiaia, Minas Gerais e Rio de Janeiro. Rodriguesia, Rio de Janeiro, v. 57, n. 1, p. 103-126, jan./abr. 2006.

PEREIRA, J. A. A.; OLIVEIRA FILHO, A. T.; LEMOS FILHO, J. P. Environmental heterogeneity and disturbance by humans control much of tree species diversity of Atlantic montane forest fragments in SE Brazil. Biodiversity and Conservation, [s. l.], v. 16, n. 6, p. 1761-1784, jun. 2007.

PIRES, G. G. et al. Influência de variáveis ambientais na comunidade arbórea de inselbergs. Cerne, Lavras, v. 20, n. 1, p. 97-104, jan./mar. 2014.

SILVA, F. C. D. S. (ed.). Manual de análises químicas de solos, plantas e fertilizantes. Brasília: EMBRAPA Informação Tecnológica; Rio de Janeiro: EMBRAPA Solos, 2009. v. 627. 624 p.

STEIN, A.; KREFT, H. Terminology and quantification of environmental heterogeneity in species-richness research. Biological Reviews, [s. l.], v. 90, n. 3, p. 815-836, aug. 2014.

TER BRAAK, C. J. F. Ordination. In: JONGMAN, R. H. G.; TER BRAAK, C. J. F.; VAN TONGEREN, O. F. R. (ed.). Data analysis in community and landscape ecology. Cambridge: Cambridge University Press, 1995. p. 91-173.

TER BRAAK, C. J. F.; PRENTICE, I. C. A theory of gradient analysis. Advances in ecological Research, Canadá, v. 18, p. 271-317, 1988.

VIEIRA, J. P. G. et al. Estudo da precipitação mensal durante a estação chuvosa em Diamantina, Minas Gerais. Revista Brasileira de Engenharia Agrícola e Ambiental, Campina Grande, v. 14, p. 762-767, jul. 2010.

ZHENQ, H.; PEIJUN, W.; JING, L.Ecological restoration of abandoned mine land in China. Journal of Resources and Ecology. [s. l.], v. 3, n. 4, p. 289-296, 2012. 